\title{
Extraction, purification and characterization of hyaluronic acid from Rooster comb
}

\author{
Sachin S. Kulkarni, Shital D. Patil and Deepali G Chavan* \\ Rajarshi Shahu College, Latur-413512, INDIA \\ Sant Ganjanan Maharaj pharmacy college Mahagaon Site Chinchewadi Kolhapur- 416503, INDIA \\ *Corresponding author. E- mail: deepa_dgc11@rediffmail.com \\ Received: August 7, 2017; Revised received: October 31, 2017; Accepted: February 5, 2018
}

\begin{abstract}
Hyaluronic acid, is extract by different procedures from various sources like pig, rabbit, oxes and human are available, but these processes have certain imitations like low yield, and also it requires the killing of these animals which is against the experimental ethics. In the present study, we have carried out the extraction of hyaluronic acid from cock's comb which was further analyzed with qualitative test, viscosity, UV absorption, endotoxin detection assay. Also, the protein contamination of extracted hyaluronic acid was determined by using SDS-PAGE of hyaluronic acid was studied for checking the protein contaminants and it was noted that there were no bands observed in the well loaded with extracted hyaluronic acid sample indicating that the final extract of hyaluronic acid is not contaminated with the protein. The extraction and purification of hyaluronic acid by using the method reported here give pure hyaluronic acid. The viscosity of extracted hyaluronic acid was found to be 2.55 poise which is economical and can be used for industrial production of hyaluronic acid having clinical applications.
\end{abstract}

Keywords: Hyaluronic acid, Osteoarthritis, protein contamination Rooster combs SDS-PAGE

\section{INTRODUCTION}

Hyaluronic acid is a long chain polysaccharide found in all mammals. It is present in loose connective tissue, skin, the eye and synovial fluid where it secreted continuously by the synovial membrane into the joint space and comprises the major macro-molecular part of the synovial fluid. It is highly concentrated at the surface of the articular cartilage and the superficial layers of the synovial membrane. In the synovial fluid, HA acts as both a lubricant and a shock absorber ( Balazs, Watson, Duff IF, and Saul 1967) Due to the meshwork it forms with aqueous solutions, it acts as a semipermeable barrier regulating metabolic exchanges between cartilage and the synovial fluid, and a viscoelastic shield around synoviocytes and adjacent nerve endings (Moreland, 2003) Through its molecular size HA hinders the free movement of lytic enzymes and inflammatory mediators, and enhances chondrocyte metabolism (Goldberg, Buckwalter 2005) Osteoarthritis is associated with a decrease in concentration and average molecular weight of native HA in synovial fluid( Pelletier and Martel-Pelletier, 1993 ). .

Hyaluronic acid has been shown to decrease dryness in the skin and offering great potential for the revitalization of the skin. It keeps skin smooth and lubricates the joints. It also plays a critical role in improving skin condition and wounds. It plays a key role both in tissue hydration and lubrication. The compound takes care of the life of skin, so the skin becomes vibrant, supple, moist and looks healthy (Mason et al., 1982). Deficiency of hyaluronic acid in human body creates problems related to joints (osteoarthritis), heart valves and eyes. Hyaluronic acid as an essential structural element in the matrix plays an important role for tissue architecture by immobilizing specific proteins (aggrecan, versican, neurocan, brevican, CD44 etc.) in desired locations within the body. It is implicated in many biological processes including fertilization, embryonic development, cell migration and differentiation, wound healing, inflammation, growth and metastasis of tumor cells and whenever rapid tissue turnover and repair are occurring ( Csoka et al., 1997). The function of Hyaluronic acid may be partly regulated and dependent on its chain length, e.g. angiogenesis is presumably induced by small HA oligosaccharides, whereas high molecular weight Hyaluronic acid exerts inhibitory effects ( West et, al., 2000). At physiological concentrations, Hyaluronic acid molecules form a random network of chains. Such a network may act as a sieve and regulate the distribution and transportation of plasma proteins. Hyaluronic acid abnormalities are common threats in connective tissue disorders (Weiss et al., 1980).

Hyaluronic acid is produced by type B lining cells of the membrane joints. It is synthesized by a class of integral membrane proteins called hyaluronan synthe- 
tase of which vertebrates have three types namely HAS1, HAS2, HAS3 (Prehm et al., 1983). These enzymes lengthen hyaluronan by repeatedly adding glucuronic acid and $\mathrm{N}$-acetyl glucosamine to the nascent polysaccharide as it is extruded through the cell membrane into the extracellular space. Hyaluronic acid is degraded by a family of enzymes called hyaluronidase. Because of its varied applications, through the present study, we are suggesting the economic way of isolating the hyaluronic acid from one of the waste material that is roosters comb of slaughter house in the form of sodium salt.

\section{MATERIALS AND METHODS}

Rooster combs and maintenance: Rooster combs are obtained from the local market immediately after the slotting of chickens. in clean plastic polypropylene bags in cold condition.

The collected rooster combs were washed under tap water to remove all dirt and blood stains followed by distilled water wash. The rooster combs were weighed, and 50 grams of rooster combs were taken in Hyaluronic acid extraction. To start with the experiment the Rooster combs were cut into small pieces with the help of clean stainless steel chopper. The collected pieces of combs were ground in an electric grinder were suspended in $100 \mathrm{ml}$ acetone and were stored at $8{ }^{\circ} \mathrm{C}$ (Swann, 1968).

After 24 hours the acetone was squeezed from the cock's comb, and additional acetone was added. This was repeated ten times at 24 hours interval. After the last extraction, the remaining acetone was evaporated in a steam of air. The weight of dried and deflated combs was found to be $8.35 \mathrm{gm}$. The dried form of combs was then extracted ten times successively with $100 \mathrm{ml}$ of $5 \%$ of Sodium acetate. Each time the viscous fluid was squeezed through several layers of cheesecloth. The final dried combs material was pure, white and fibrous in appearance. This dried material was precipitated with sodium saline citrate. This mixture was squeezed with cheesecloth. At last the obtained precipitate was purified by using the procedure suggested by (Meyer and Palmer, 1979).

Removal of pyrogen from the hyaluronic acid: From the purified extract Pyrogen was removed by centrifuging it at $150000 \mathrm{rpm}$ for 20 minutes and the sediment was removed. The sodium hyaluronate in the form of the gel after taking out the supernatant is washed by adding $70 \%(\mathrm{v} / \mathrm{v})$ water-soluble organic solvent. By this operation, the sodium hyaluronate turns from gel to powder. Further, the extract was subjected to vacuum drying whereby sodium hyaluronate is obtained. It was reported that extraction by this method removes the pyrogens at a level of 0.0015 to $0.003 \mathrm{EU} / \mathrm{mg}$ or less (Prescott et al., 2003). The obtained powdery sodium hyaluronate was allowed to dry under aseptic conditions and was confirmed by using the confirmative test.

Confirmative test: The obtained sample of hyaluronic acid was mixed with toludine blue (Ramalingam et al., 1970). From the observation (green color formation) it was confirmed that the obtained sample consists of hyaluronic acid.

Identification of hyaluronic acid: One of the methods used for identification of Hyaluronic acid is viscosity (Eiji Shimada et al., 1975) which was done by using Oswald's viscometer. Hyaluronic acid is identified by observing the viscosity of extracted liquid (Chester, 1964). Water is used as a standard whose viscosity is known.

UV spectral analysis of extracted hyaluronic acid: Hyaluronic acid absorbs maximally at 190-200 nm wavelength (Lapcik, 1992). To confirm the obtained sample is hyaluronic acid the pattern of absorption of hyaluronic acid was studied using Spectrophotometer.

Endotoxin detection assay of extracted hyaluronic acid: Before starting with the endotoxin detection assay, all glassware was soaked overnight in cleaning agent, E-Toxa-Clean (Sigma), rinsed in pyrogen-free water and oven dried at $300{ }^{\circ} \mathrm{C}$. Finally, the purified extract of hyaluronic acid from Rooster comb hyaluronan was analyzed for endotoxin, using the Limulus amoebocyte lysate assay (E-Toxate, Sigma).

As hyaluronic acid is a polysaccharide, while following the purification protocols of hyalronic acid for its clinical applicaons, proteins act as one of the unwanted and major contaminants. Thus, it is important to have a hyaluronic acid at the end of purification without protein contamination. In the present study, we have checked the protein contamination in the final extract of hyaluronic acid by using SDS PAGE ( He, 2011).

\section{RESULTS AND DISCUSSION}

Hyaluronic acid is present in appreciable quantities in the cock's comb and can be isolated in relatively pure form in present study The obtained hyaluronic acid was confirmed by using qualitative confirmative test / Toluidine blue test which was positive that is in blue

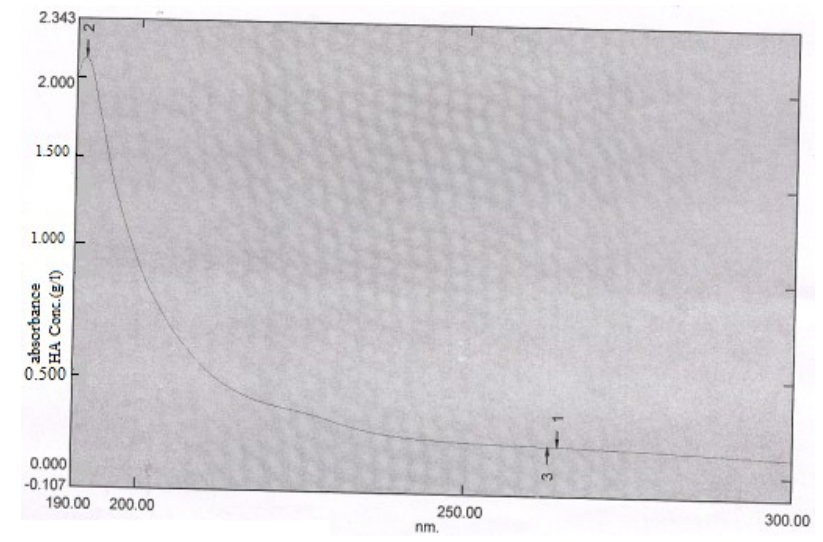

Fig. 1. UV spectral analysis of extracted hyaluronic acid showing absorption maxima at $191.4 \mathrm{~nm}$. 


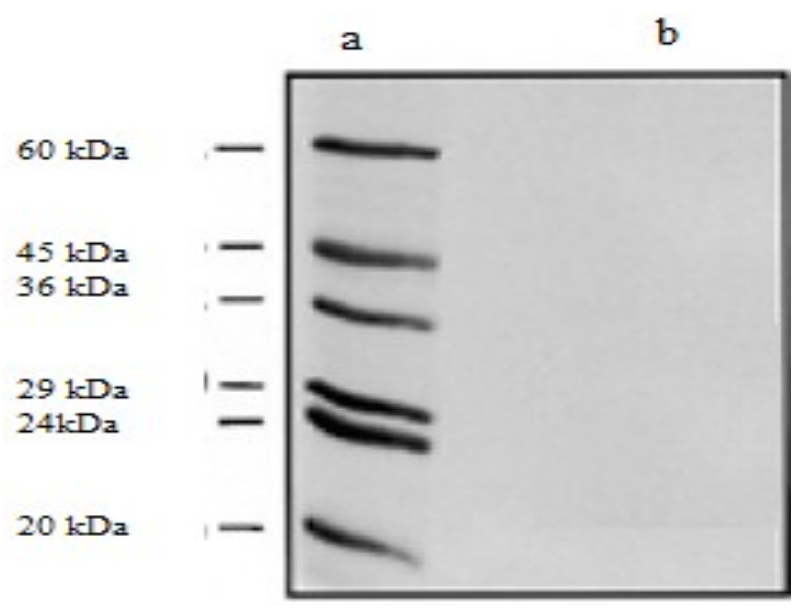

Fig. 2. SDS PAGE of hyaluronic acid extract (a): Molecular weight markers,-bands show contamination with protein( $b$ ) No bands showing hyaluronic acid extract in pure form.

colour formation is same as (Ramalingam et al., 1970). The viscosity was used for the base of identification of Hyaluronic acid. The viscosity of extracted hyaluronic acid was found to be 2.55 poise by using water as a standard (Eiji Shimada et al., 1975) so now it is partially purified. Thus, it is expected to check the purity of extracted hyaluronic acid for which UV absorption where the extract Hyaluronic acid sample showed maximum absorption at $191.4 \mathrm{~nm}$ (Fig.1). Similar results of absorption of hyaluronic acid were reported by Lapcik (1992) where they got maximum absorption at $200 \mathrm{~nm}$ of Hyaluronic acid copper (II) complexes spectroscopic characterization (UV spectra of pure hyaluronic acid solution before and after addition of copper ion) .In the endotoxin detection assay we check the protein contamination in the final extract of hyaluronic acid by using SDS PAGE (Fig. 2) bands are observed it is contaminated by protein(molecular weight markers) no bands are observed hyaluronic acid extract is in pure form same as a result as of (Laemmli, 2011).

\section{Conclusion}

The results obtained shows that the applied methodology is effective for extracting and purifying hyaluronic acid. The quantitative and qualitative analysis shows that viscosity of extracted hyaluronic acid was found to be 2.55 poise and SDS PAGE (Laemmli, 2011). of hyaluronic acid extracted from showed that no bands observed in well loaded with extracted hyaluronic acid sample indicating that the final extract of hyaluronic acid is not contaminated with the protein gives pure hyaluronic acid. Thus the hyaluronic acid obtained from chicken combs may be used as a co-product from the poultry industry for research and clinical applications

\section{REFERENCES}

Balazs, (1979). patent No. 4141937, 514/54, Ultrapure hyaluronic acid and the use of hyaluronic acid
Cassaro, C.M. and Dietrich, C.P. (1977). Distribution of sulfated mucopolysaccharides in invertebrates. $J$ Biol Chem., 252(7):2254-2261.

Chester, T. Stafford, William, Niedermeier, Howard, Holley, L. and Ward Pigman (1964). Studies on the Concentration and Intrinsic Viscosity of Hyaluronic Acid in Synovial Fluids of Patients with Rheumatic Diseases Ann. rheum. dis. 23:152-157.

Csoka T.B., Frost, G.I., Stern, R. (1997). Hyaluronidases in tissue invasion. Invasion Metastasis. 17:297- 311.

Eiji Shimada and Go Matsumura. (1995). Viscosity and Molecular Weight of Hyaluronic Acids. J Biochem 78 (3): 513-517.

Lapcik, L., Ch. Dammer and M. Valko. (1992). Hyaluronic acid copper(II) complexes: spectroscopic characterization. Journal colloid and polymer science, 27(10):10491052.

Mason, R.M., d'Arville, C., Kimura JH, Hascall VC. (1982). Absence of covalently linked core protein from newly synthesized hyaluronate. Biochem J., 207(3):445-457.

Prehm P. (1983). Synthesis of hyaluronate in differentiated teratocarcinoma cells. Mechanism of chain growth. Biochem J., 211(1):191-198.

Ramalingam, K. and Ravindranath, M. H. (1970). Histochemical significance of green metachromasia to Toluidine blue. Histochemistry and cell biology. 24(4): 322327.

Swann, D.A. (1968). Studies on hyaluronic acid. I. The preparation and properties of rooster comb hyaluronic acid. Biochim Biophys Acta., 156(1):17-30.

Swann, D.A. (1968). Studies on hyaluronic acid. II. The protein component(s) of rooster comb hyaluronic acid. Biochim Biophys Acta., 160 (1):96-105.

Weiss, C, Balazs, E.A., St. Onge, R., Denlinger, J.L. (1981). Clinical studies of the intra articular injection of HealonR (sodium hyaluronate) in the treatment of osteoarthritis of human knees. Osteoarthritis symposium. Palm Aire, Fla., 20-22.

West, D.C., Chen, H. (2000). Is hyaluronan degradation an angiogenic/ metastatic switch? New Frontiers in Medicinal Sciences: Redefining Hyaluronan; Elsevier Science, 77-86.

Balazs EA, Watson D, Duff IF, Saul R (1967) Hyaluronic acid in synovial fluid. I. Molecular parameters of hyaluronic acid in normal and arthritic human fluids. Arthritis Rheum, 10: 357-376.

Moreland LW (2003) Intra-articular hyaluroran and hylans for the treatment of osteoarthritis: mechanisms of action. Arthritis Res. Ther., 5: 54-67.

Goldberg, V.M., Buckwalter, J.A. (2005). Hyaluronans in the treatment of osteoarthritis of the knee: evidence for disease modifying activity. Osteoarthritis Cartilage, 13: 216- 224.

Pelletier, J.P., Martel-Pelletier, J. (1993). The pathophysiology of osteoarthritis and the implication of the use of hyaluron and hylan as therapeutic agents in viscosupplementation. J. Rheumatol Suppl., 39: 19-24.

He, F. (2011). Laemmli-SDS-PAGE. Bio-protocol Bio101: e80. DOI:10.21769/BioProtoc.80.

Meyer, K., Palmer, J. W. (1979). Extraction from rooster combs and purification: EP 138572.

Prescott A. L. (2003). Method for purifying high molecular weight hyaluronic acid. USP 6660853. 